\title{
Phase diagram for coherent vortex formation in the two-dimensional inviscid fluid in circular geometries
}

\author{
Peilong Chen and M. C. Cross \\ Condensed Matter Physics 114-36, California Institute of Technology, Pasadena, California 91125
}

(Received 26 October 1993)

\begin{abstract}
We calculate the equilibrium states of a two-dimensional inviscid fluid in disk and annular geometries using the mean field equations that respect all conservation laws of the Euler equations. Axisymmetric vorticity distributions and their bifurcations to asymmetric solutions are calculated for a wide range of system parameters. Approximate zero-temperature compact vortices are also constructed and compared to symmetric states. From these results, the parameter ranges leading to the formation of a coherent vortex by the long time evolution from given initial conditions can be predicted without knowledge of the intervening dynamics. Applications to models of Jupiter's great red spot and experiments on onecomponent plasmas in a magnetic field are discussed.
\end{abstract}

PACS number(s): 47.20. $-\mathrm{k}, 05.20 . \mathrm{Gg}, 52.25 . \mathrm{Kn}, 92.90 .+\mathrm{x}$

\section{INTRODUCTION}

Long-time solutions of the inviscid two dimensional fluid equations (the Euler equations) have been suggested to be important in a number of interesting physical situations. These include the formation of coherent structures in planetary atmosphere (Jupiter's red spot is perhaps the most famous example), two-dimensional large Reynolds number turbulence, and the dynamics of a onecomponent plasma in a strong magnetic field. Recently two groups [1,2] have proposed that a new statistical mechanical formulation, a consistent description of the Euler system respecting the infinity of conserved quantities in this system, may be used to investigate these states without the numerically expensive process of solving the long time evolution of the dynamical equations.

In this paper we use these ideas to investigate the equilibrium states of the two-dimensional inviscid fluid in a disk and in an annulus-geometries that are relevant to these physical systems. In particular, we use the relative simplicity of the statistical mechanical approach to broadly survey the range of system parameters for which the formation of coherent blobs of vorticity is to be expected.

The great red spot of Jupiter is a remarkable phenomenon that has excited astronomers and physicists since its first observation. We can now say that this structure has remained stable for over three hundred years, a very long time compared to the time scale of the small features of the flow in the turbulent background that usually last for a few hours or a day. Although dissipation and driving by the absorption of small scale vorticity must clearly be involved in a complete theory, the tendency of an inviscid two-dimensional fluid to form coherent spots of vorticity may be an important aspect of the problem, so that an equilibrium description may be used. Different models have been proposed to study the outer layer of planetary atmospheres [3-8]; for example, the coherent vortices in the quasigeostrophic regime and the Rossby solitons in the intermediate geostrophic re- gime. Many time-dependent numerical simulations and experiments on shallow water systems have suggested the formation of spotlike coherent structures. In particular, we make careful comparison with the dynamical simulations of the two-dimensional Euler equation in an annulus $[6,9]$ where coherent vortices are formed under certain conditions of the background shear flow.

Coherent vortices are important in two-dimensional turbulence as well. For the Navier-Stokes equations with small viscosity, many high resolution dynamic integrations have been done recently [10-15]. There is also a recent experimental realization [16]. These results confirm the formation of coherent vortices in the long time evolution, but there is still some debate on the decay rate of the turbulence. Some papers favor the selective decay hypothesis for the fluid [13-15]. Another decay mechanism has also been proposed $[17,18]$ which assumes the conservation of the peak vorticity value of the system. Smith [19] has suggested that a statistical mechanics investigation of a vortex system can serve as a model and help to understand the relaxation of two-dimensional turbulence at very large Reynolds numbers.

The equilibrium states of the two-dimensional Euler equation can also be compared to the results of experiments on a pure electron plasma in strong magnetic fields [20]. If the electron plasma is approximated by the guiding center model, the governing equation is the Euler equation. The experiments can be set up with arbitrary initial electron distributions, and the dynamics and final states can be studied. It will then be interesting to compare the final distributions of electrons with the equilibrium descriptions of the Euler equation.

The equilibrium description of two-dimensional inviscid fluid flow was first suggested by Onsager [21]. He used a system of identical point vortices to model the ideal fluid and applied statistical mechanics to the system using the Hamiltonian $H=-\sum_{i \neq j} \omega_{i} \omega_{j} G\left(\mathbf{r}_{i}, \mathbf{r}_{j}\right)$, first written down by Kirchhoff [22], with $G$ the Green's function for the Laplacian. Onsager argued that in a bounded system negative temperatures will be found at high 
enough system energy due to the finite nature of the phase space, which here is the same as the system volume.

The statistical equilibrium equation for a point vortex system is the sinh-Poisson equation [23]. Calculations of the equilibrium states have been done under various conditions [23-28] and negative temperatures appear in many of these solutions, with the appearance of coherent vortices. However, in the point vortex model, where the vorticity distribution is assumed to be a sum of many singular $\delta$ functions, the infinite number of conserved quantities in the two-dimensional Euler flow, $\int_{\Omega} d \mathbf{r} \omega^{n}(\mathbf{r})$ with $n$ any integer and $\Omega$ the total volume, are not defined.

Equations for the statistical equilibrium states of the Euler equation respecting all the conserved quantities have been formulated in Refs. [1], [2], and [29]. In these papers the vortex distribution is written in terms of a vorticity distribution function $n(r, \sigma)$, with $r$ the position vector and $\sigma$ the vorticity level. This formulation eliminates the singularity problem of the point vortex model and will give a consistent description of the twodimensional Euler flow.

In this paper we use the vortex formulation respecting all the conserved quantities to find the nature of the equilibrium states in circular geometries resulting from initial conditions described by two vorticity levels. Although the restriction to two levels is a simple model, it preserves the essence of the theory, namely, the infinite number of conserved quantities, and corresponds to the initial conditions chosen by previous workers $[6,9]$. First the axisymmetric solutions with only radial dependence for the vorticity distribution are computed. The solutions are described by curves in the energy-entropy or energytemperature planes for fixed values of the other parameters characterizing the system (the total vorticity $Q$, maximum vorticity level $q$, angular momentum $M$, and, in the case of an annular geometry, the circulation around the inner boundary $-2 \pi C$ ). We identify the tendency towards coherent vortex formation as a bifurcation of the axisymmetric solution towards nonaxisymmetric ones. Where this bifurcation is continuous, we can accurately calculate the energy at which the bifurcation occurs as a function of the other parameters by a linear stability analysis. For other parameter values the bifurcation must be discontinuous (first order), and we do not have good estimates in this case. Large energy, nonaxisymmetric, states in the disk are calculated approximately by a variational approach. Finally the zero-temperature nonaxisymmetric vorticity distributions and their properties are approximately calculated. Together with the linear stability analysis this sets limits (particularly on the circulation parameter $C$ ) for which coherent vortex formation is expected. We compare our results with previous results from numerical simulations of the long time evolution.

\section{VORTEX STATISTICAL EQUILIBRIUM}

For a two-dimensional incompressible ideal fluid, the equation of mass conservation becomes $\nabla \cdot u(r)=0$, with $\mathbf{u}(\mathbf{r})$ the velocity field. We can define the stream function $\psi(\mathbf{r})$ to describe the fluid such that $\mathbf{u}(\mathbf{r})=\nabla \times \psi \hat{\mathbf{z}}$ $\equiv(\partial \psi / \partial y,-\partial \psi / \partial x)$. Introducing the stream function automatically satisfies the incompressibility condition. The equation of motion of the fluid is

$$
\frac{\partial \mathbf{u}}{\partial t}+(\mathbf{u} \cdot \nabla) \mathbf{u}=-\nabla p
$$

Here $p$ is the fluid pressure. Taking the curl of the above equation and defining vorticity $\omega(\mathbf{r}) \widehat{\mathbf{z}}=\nabla \times \mathbf{u}(\mathbf{r})$ yields the equation of motion

$$
\frac{\partial \omega}{\partial t}+(\mathbf{u} \cdot \nabla) \omega=0
$$

This equation implies that there are infinite numbers of conserved quantities $\int_{\eta(t)} f(\omega(\mathbf{r})) d^{2} r$ for any path $\partial \eta(t)$ moving with the fluid and $f$ an arbitrary function. The Hamiltonian of the system is $H=\frac{1}{2} \int|\mathbf{u}(\mathbf{r})|^{2} d^{2} r$. Under the Hamiltonian and the constraints of the conserved quantities, the statistical equilibrium states of the fluid are determined self-consistently by $[1,29]$

$n(\mathbf{r}, \sigma)=\frac{\exp \{-\beta[\sigma(\psi(\mathbf{r})-h(\mathbf{r}))-\mu(\sigma)]\}}{\int_{-\infty}^{\infty} d \sigma^{\prime} \exp \left\{-\beta\left[\sigma^{\prime}(\psi(\mathbf{r})-h(\mathbf{r}))-\mu\left(\sigma^{\prime}\right)\right]\right\}}$,

where the stream function $\psi(r)$, with accompanying vorticity $\omega(\mathbf{r})$, is determined by $n$,

$$
-\nabla^{2} \psi(\mathbf{r})=\omega(\mathbf{r})=\int_{-\infty}^{\infty} d \sigma \sigma n(\mathbf{r}, \sigma) .
$$

The function $\mu(\sigma)$ is determined by the $g(\sigma)$ constraint,

$$
g(\sigma)=\int d^{2} r n(r, \sigma),
$$

with $g(\sigma)$ the constant vorticity distribution fixed by initial conditions. The energies of the system may be calculated from

$$
\begin{aligned}
E & =\frac{1}{2} \int d^{2} r|\mathbf{u}(\mathbf{r})|^{2} \\
& =\frac{1}{2} \int d^{2} r \psi \omega+\frac{1}{2} \oint_{\text {boundary }} \psi(\nabla \times \psi) \cdot d 1,
\end{aligned}
$$

and the inverse temperature $\beta$ is determined by fixing the energy. The function $h(r)$ may be used to account for the external fields and other conserved quantities in geometries of special symmetry.

We solve the equations for a choice of the function $g(\sigma)$ such that there are two levels of vorticity which may be chosen to be $\sigma=q$ and $\sigma=0$ on fractional areas $\alpha$ and $1-\alpha$, respectively:

$$
g(\sigma)=(1-\alpha) \delta(0)+\alpha \delta(q) \quad 0 \leq \alpha \leq 1 .
$$

In the equilibrium state the vorticity distribution $\omega(\mathbf{r})$ will typically take on a continuum of values, always bounded by the value $q$. Because both disk and annulus which we consider are rotationally symmetric, $h(r)$ includes a Lagrange multiplier term $\Omega r^{2}$ to impose conservation of angular momentum. The entropy of the system is calculated by

$$
S=-\int d^{2} r\left[\frac{\omega}{q} \ln \left[\frac{\omega}{q}\right)+\left(1-\frac{\omega}{q}\right) \ln \left[1-\frac{\omega}{q}\right)\right] .
$$


With the given $g(\sigma)$ and $h(r)$, the equation of vorticity distribution becomes

$$
\nabla^{2} \psi(\mathbf{r})=-\omega(\mathbf{r})=\frac{-q}{1+\exp \left\{-\beta\left[q \psi(\mathbf{r})+\Omega r^{2}-\mu\right]\right\}} .
$$

Here $\mu$ and $\Omega$ are constants to be determined by conservation of total vorticity and angular momentum,

$$
\begin{aligned}
& Q=\alpha q V=\int \omega d^{2} r, \\
& M=\int r^{2} \omega d^{2} r .
\end{aligned}
$$

The true fluid angular momentum is

$$
\int r v_{\theta} d^{2} r=-\frac{1}{2} \oint_{\text {boundaries }} r^{3} \frac{\partial \psi}{\partial r} d \theta-\frac{1}{2} \int r^{2} \omega d^{2} r
$$

in a circular geometry. It can be easily shown from the Euler equations that the circulation on every boundary $\oint_{\text {boundary }} \mathrm{u} \cdot d \mathbf{l}$ is a constant. This fixes the first term in the angular momentum for circular geometries, so that only the second term is used as the angular momentum $M$. Equation (2) is the distribution of an ideal hard core gas in an external potential with the size of the hard core depending on $\alpha$, the fractional filling of the vorticity $q$ regions.

We calculate the vorticity distributions in two geometries, a unit disk and an annulus with $r_{\text {inner }}=1$ and $r_{\text {outer }}=2$. Because the fluid cannot penetrate the boundary, we have $u_{r}=(1 / r) \partial \psi / \partial \theta=0$ on the boundary, and so the stream function will be constant on every boundary. In the disk the boundary conditions are that the stream function is regular at $r=0$ and may be taken to be zero at the boundary. The stream function in the annulus can be set to zero on the outer boundary. Thus in the annulus there may be a conserved circulating flow in addition to the flow induced by the vorticity within the annulus. Although the constant value of the stream function on the inner disk will vary depending on the vorticity distribution in the annulus, this circulation will not change. We therefore define $C$ such that, with constant $\psi(r=1, \theta)$,

$$
\left.\int \frac{\partial \psi}{\partial r}\right|_{r=1} d \theta=2 \pi C \text {. }
$$

Note that $-2 \pi C$ will then be an effective vorticity at the center of the inside cylinder.

The nonzero value of $C$ also produces a background shear in the system. For zero vorticity in the annulus, $\omega=0$ with $C=\left.(\partial \psi / \partial r)\right|_{r=1}, u_{\theta}=-C / r$, and so the shear becomes

$$
\sigma \equiv r \frac{\partial}{\partial r}\left[\frac{u_{\theta}}{r}\right]=\frac{2 C}{r^{2}} .
$$

The shear $\sigma$ has the same sign as $C$.

\section{AXISYMMETRIC SOLUTIONS}

First we consider the axisymmetric solutions. With no $\theta$ dependence, Eq. (2) takes the form

$$
\begin{aligned}
\frac{1}{r} \frac{d}{d r}\left(r \frac{d \psi_{0}(r)}{d r}\right) & =-\omega_{0}(r) \\
& =\frac{-q}{1+\exp \left\{-\beta\left[q \psi_{0}(r)+\Omega r^{2}-\mu\right]\right\}}
\end{aligned}
$$

We numerically integrate the equation using the boundary conditions mentioned in the previous section. By using a standard root finding procedure, values of $\beta, \Omega$, and $\mu$ are determined by fixing the total energy, total angular momentum, and total vorticity. The solutions for the annulus are shown in Fig. 1.

The first two rows of Fig. 1 show the states of the system characterized by the energy, entropy, and inverse temperature $\beta$ for $Q=2, q=1, M=4$ (so that $\alpha=2 / 3 \pi$ ), and various values of the circulation $C$. The energies are bounded for both small and large values. Contrary to the point vortex system, where the vorticity is unbounded so that energies can go to infinite values, the maximum vorticity here is finite due to the hard core property. The two limiting energy states corresponding to $\beta$ going to $\pm \infty$ are clearly seen in the second row of Fig. 1. The systems have maximum entropy when $\beta$ is zero, consistent with the thermodynamic relation $\beta=\partial S / \partial E$. The entropies usually go to zero when $\beta$ goes to $\pm \infty$, or equivalently when the temperature goes to zero. This is because in these low and high energy limits the vorticity will saturate at value $q$ in a finite region of space and then Eq. (1) will give zero entropy. The vorticity distributions in these limits are shown in the bottom row of Fig. 1. The distributions can be understood as follows. In (ii) and (iii)

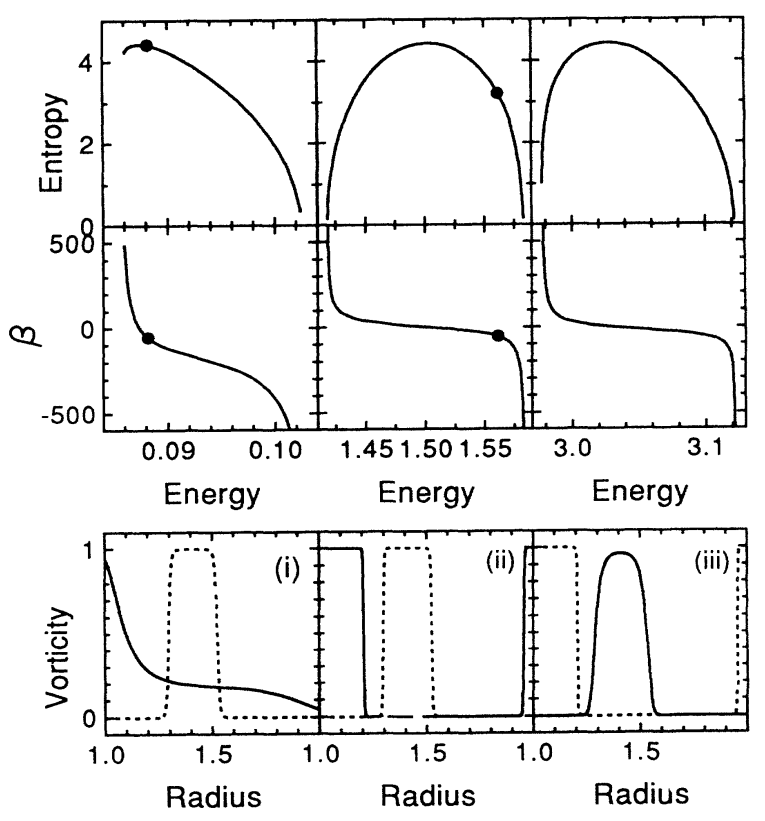

FIG. 1. Axisymmetrical solutions in the annulus with total vorticity $Q=2$, angular momentum $M=4$, and initial vorticity level $q=1$. The three columns are with circulation $C$ equal to 0 , 1 , and -1 , respectively. On the bottom row showing the vorticity distributions, solid lines are for large positive inverse temperature $\beta$; dashed lines for large negative $\beta$. The solid circles are the bifurcation points. 
the dominating forces are the interactions between the effective vorticity at the center and the vorticity distribution in the annulus. With negative values of $\beta$, the interaction is repulsive in (ii) and attractive in (iii), and the vorticity distributions (dashed lines) become a top hat distribution centered away from the walls in (ii) and two distinct regions on the walls in (iii). The reason for the two regions in (iii) is that the circulation inside attracts vorticity to the inner wall, with the rest sent to the outer wall to conserve angular momentum. With positive values of $\beta$, the interactions change sign and the distributions (solid lines) just reverse the situation for the dashed lines. In (i) with zero circulation inside, there are only the self-interactions between the vortices. The dashed line distribution comes from the self-attraction of the vortices at the negative inverse temperature. The reason for the distribution (solid line) at positive $\beta$ is explained in the next paragraph.

The results for the disk geometry are shown in Fig. 2. They are similar to the case in the annulus with no circulation inside. The interesting case is when $\beta \rightarrow \infty$. In this limit, the system becomes equivalent to an electrostatic problem $\nabla^{2} \psi=0$ with an external potential $-\rho r^{2}$, imposed by the constraint of total angular momentum. This potential can also be produced by a uniform charge distribution $-\rho$ in the disk. Thus, to minimize the energy, the vortices will tend to neutralize the uniform negative charge distribution. This will produce a step vorticity distribution and the constant vorticity not equal to $q$ im-
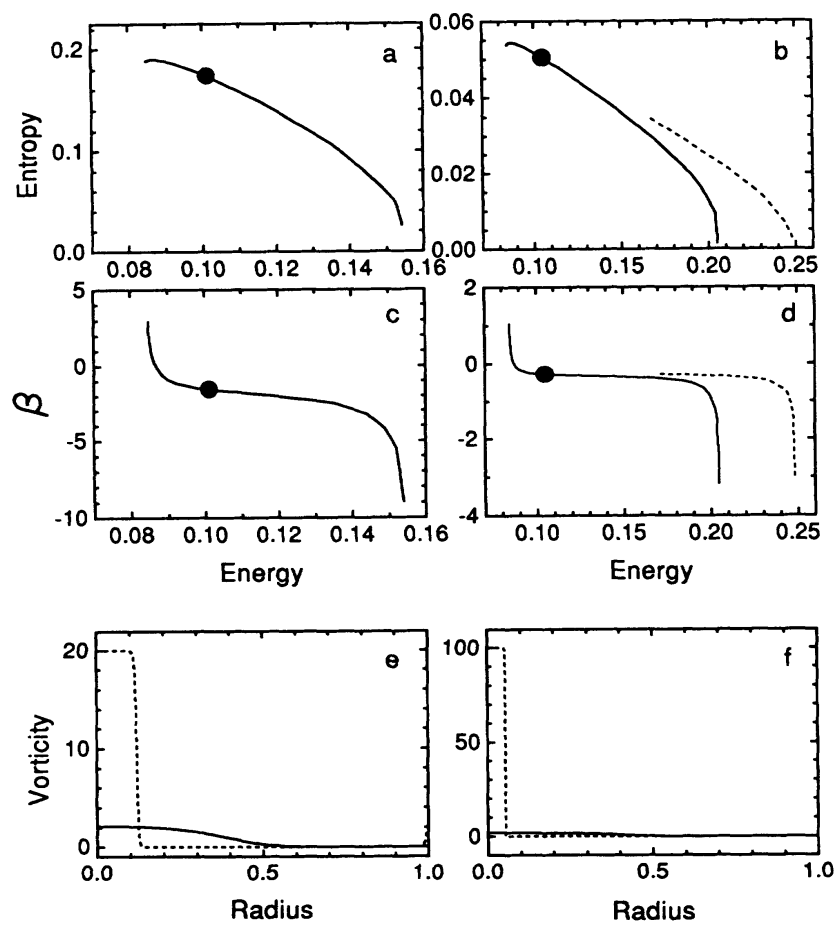

FIG. 2. Axisymmetrical solutions in the disk with $Q=1$ and $M=0.1$. The two columns are with $q=20$ and $q=100$, respectively. On the bottom row, solid lines for large positive $\beta$; dashed lines for large negative $\beta$. The solid circles are the bifurcation points. The dashed lines in entropy-energy and $\beta$-energy spaces are for the compact spot approximation. plies a nonzero entropy at zero temperature, as seen in Figs. 2(a) and 2(b). Because numerically it is hard to approach this limit, the solid lines in Figs. 2(e) and 2(f) for the vorticity distribution with large $\beta$ do not show true steps.

\section{LINEAR PERTURBATION}

At high energies or large negative $\beta$ the vortex system is likely to be an asymmetrical distribution instead of a symmetrical one. In a Monte Carlo simulation [1], the high energy state for the vortex distribution is indeed shown to be localized. A neutral vortex system dynamical simulation also shows the formation of a single vortex $[6,9]$. This behavior has also been studied in the point vortex model [27,28]. Here we use the formulation respecting all conserved quantities to study the bifurcation. Qualitatively speaking at large negative $\beta$ the effects of the self-attraction between vortices are stronger. This self-attraction will eventually break the axisymmetric distribution and a bifurcation will occur. To study the bifurcation of the axisymmetric vortex distribution to asymmetric ones, we now add a small perturbation $\delta \psi(r, \theta)$ and $\delta \omega(r, \theta)$ to the axisymmetric equilibrium states $\psi_{0}(r)$ and $\omega_{0}(r)$ :

$$
\begin{aligned}
\psi(\mathbf{r}) & =\psi_{0}(r)+\delta \psi(r, \theta) \\
& =\psi_{0}(r)+\left[\delta \hat{\psi}(r) e^{i m \theta}+\text { c.c. }\right], \\
\omega(\mathbf{r}) & =\omega_{0}(r)+\delta \omega(r, \theta) \\
& =\omega_{0}(r)+\left[\delta \hat{\omega}(r) e^{i m \theta}+\text { c.c. }\right] .
\end{aligned}
$$

Here we expand $\delta \psi$ and $\delta \omega$ in angular modes. $\delta \omega(r, \theta)$ does not change the total vorticity for $m>0$. Substituting the expressions into Eq. (2) and keeping only the first order terms in $\delta \hat{\psi}$ and $\delta \hat{\omega}$, we get the following equations for the perturbed quantities:

$$
\begin{aligned}
& {\left[\frac{1}{r} \frac{d}{d r}\left[r \frac{d}{d r}\right]-\frac{m^{2}}{r^{2}}+\beta \omega_{0}(r)\left[\omega_{0}(r)-q\right]\right] \delta \hat{\psi}(r)=0,} \\
& \beta \omega_{0}(r)\left[\omega_{0}(r)-q\right] \delta \hat{\psi}(r)=\delta \hat{\omega}(r) .
\end{aligned}
$$

The boundary conditions are as follows: in the disk $\delta \hat{\psi}$ is equal to zero at $r=0$ and $r=1$, and in the annulus we have $\delta \hat{\psi}(r=1)=\delta \hat{\psi}(r=2)=0$. Equation (3) is an eigenvalue equation for $\delta \hat{\psi}(r)$. The solution of $\delta \hat{\psi}(r)$ and $\delta \hat{\omega}(r)$ will only exist for a certain value of $\beta$. This defines the bifurcation point of the axisymmetric states. We numerically integrate Eq. (3) and find the values of $\beta$ which give solutions of $\delta \hat{\psi}(r)$ with the required boundary conditions. The $m=1$ bifurcation points, which have a lower energy than the ones for $m \geq 2$, are marked on the system state curves as solid circles in Figs. 1 and 2 [30]. Note that there is no bifurcation for $C=-1$ in the annulus.

As expected, all the bifurcations happen at negative inverse temperatures. From the form of the system energy $H=\frac{1}{2} \int|\mathbf{u}(\mathbf{r})|^{2} d^{2} r$, the bifurcation energy will scale with the square of the total vorticity $Q$. The energy will also depend on the circulation within the inside cylinder $-2 \pi C$, total angular momentum $M$, and $\alpha$, which 
characterizes the size of the hard core. So we can expect the quantity $E / Q^{2}$ be a function of $C / Q, M / Q$, and $\alpha$. This has been verified by our calculations using different values of $Q$. Figure 3 plots the $m=1$ bifurcation energy $\left(E-E_{\min }\right)$ as a function of $C$, with $M=4, Q=2$, and $\alpha=2 / 3 \pi$, with $E_{\min }$ the minimum possible energy for the axisymmetric distribution. The dashed line marks the maximum possible energy $E_{\max }-E_{\min }$ for the axisymmetric solution. This limitation comes from the hard core property. The bifurcations exist for $C$ between two critical values $C_{c}^{\text {lower }}=-0.2741$ and $C_{c}^{\text {upper }}=2.521$. In this region the symmetrical distribution will be unstable to a nonaxisymmetric perturbation if the system energy is larger than that of the bifurcation point. A second bifurcation point also occurs for $C$ near $C_{c}^{\text {lower }}$ and $C_{c}^{\text {upper }}$. These lines divide the $E-C$ parameter space into two regions. In the upper region, we have unstable symmetrical solutions and presumably an asymmetrical coherent vortex will be the long-time state. Qualitatively similar diagrams are found when different values of $\alpha$ and $M$ are used. The value of $C_{c}^{\text {upper }}$ is quite sensitive to changes in $\alpha$ and $M$, but $C_{c}^{\text {lower }}$ is less sensitive. We suggest that this is because for positive $C$ the symmetric vorticity distribution at the bifurcation is strongly peaked with a peak value growing with $C$ and with a position determined by $M$. The driving force in the bifurcation may be ascribed to the increased vorticity interaction due to its larger peak value in the asymmetric coherent vortex. As $C$ increases, however, the vorticity in the symmetric ring also approaches saturation, so that a maximum value $C_{c}^{\text {upper }}$ for which the bifurcation occurs is to be expected. Since the approach to saturation in the symmetric state will depend strongly on $\alpha$, the rapid dependence of $C_{c}^{\text {upper }}$ on this quantity is accounted for. In addition, as $M$ approaches its maximum or minimum value, the peak vorticity will be increased as the tails of the vorticity distribu-

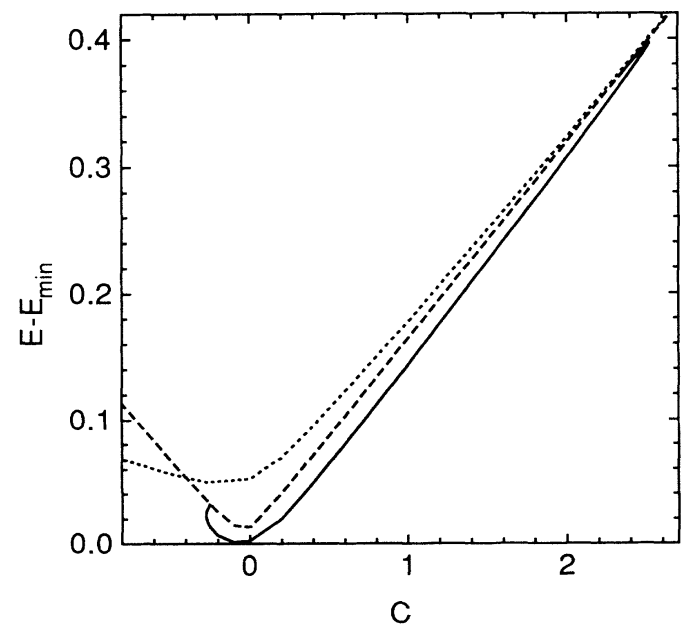

FIG. 3. Phase diagram for asymmetric solutions. The solid line is the bifurcation line for the $m=1$ mode in the annulus with $Q=2, M=4$, and $q=1$. The dashed line is the maximum energy for the axisymmetric distribution. The dotted line is the energy for the variational calculation of the maximum energy asymmetric solution. tion with radius are truncated by the walls, producing a rapid variation of $C_{c}^{\text {upper }}$ with this parameter as well. On the other hand, at $C_{c}^{\text {lower }}$ the symmetric vorticity distribution is not strongly peaked, the maximum value of the vorticity is well below saturation, and so a much weaker dependence of $C_{c}^{\text {lower }}$ on $\alpha$ and $M$ is expected.

\section{COMPACT SPOT APPROXIMATION}

In the disk, an approximate solution of the mean field equation can be computed when the vortices are close packed at large energy. This is a straightforward generalization of the point vortex calculation in Refs. [27] and [28]. Assume that the vortex is localized at the position D away from the wall and the size of the localization of the vortex is much smaller than $D$. Write the stream function $\psi(\mathbf{r})$ as $\psi_{I}(\mathbf{r})+\psi^{\prime}\left(\mathbf{r}^{\prime}\right)$, with $\psi_{I}(\mathbf{r})$ the image potential of a point vortex with strength equal to the total vorticity $Q$ at $D$, and $\mathbf{r}^{\prime}=\mathbf{r}-\mathbf{D}$. After Taylor expansion of $\psi_{I}(r)$ about $\mathbf{D}$, the governing equation involves only $r^{\prime}$ and can easily be numerically integrated. Since the approximation is more accurate for a more localized distribution, we calculate the case in the disk with $q=100$.

The results are shown in Figs. 2(b) and 2(d) as dashed lines. The branch goes to a limiting energy and zero entropy when $\beta$ goes to infinity. This is the state in which the distribution of the vorticity is a spot with constant value $q$. For a given energy above the bifurcation point, the asymmetrical solution always has a higher value of the entropy and so to maximize the free energy the asymmetrical distributions will be the statistical equilibrium states. Although the compact spot approximation will break down at smaller energies, the intersection of the extrapolation of the dashed lines in Figs. 2(b) and 2(d) with the solid line is consistent with the bifurcation points marked by the solid circles.

\section{ZERO-TEMPERATURE STATES}

A second way of estimating the tendency towards coherent vortex formation in the annular geometry is a comparison of the energy of a zero-temperature nonaxisymmetric state, calculated approximately, with the energy of the axisymmetric zero-temperature state. In the point vortex model, when $\beta \rightarrow-\infty$, the vorticity distributions become singular and the energy diverges. The point vortex model clearly fails in this limit and cannot give the properties of the limiting states. However, in the present model, we can have a finite vorticity distribution when $\beta \rightarrow-\infty$. In this limit, $\omega(\mathbf{r})$ will generally be constant at $q$ inside the spot and zero elsewhere. Both entropy $S$ and temperature $T$ are equal to zero for this distribution.

To calculate their properties we approximate the coherent vortex as a sector of constant vorticity with span angle $\phi$, inner radius $a$, and outer radius $b$. The total vorticity and total angular momentum give two constraints on the values of $\phi, a$, and $b$, leaving one number to be chosen variationally. With the known vorticity distribution the stream function calculation is analogous to the electrostatic problem, $\nabla^{2} \psi=-\omega$. Using the Green 
function method we have calculated the energies of the system for various values of span angle $\phi$.

On choosing the span angle $\phi$ as the free parameter, the typical curve for the system energy $E$ versus $\phi$ is in Fig. 4. For most parameter values there is a maximum energy for a particular value of the span angle or the maximum occurs at the boundary of the possible range of the span angle. Since we are at negative temperatures, the thermodynamic equilibrium state will be the one with the largest free energy $F$. If the maximum energy is larger than the energy calculated from the axisymmetric solution at the negative infinite $\beta$ limit, the vortex spot will be the preferred state. To explain this, consider a symmetrical distribution in the limit. The vorticity is a constant value $q$ in a circular belt. Both the temperature $T$ and the entropy $S$ are equal to zero, so the free energy $F$ is the same as the system energy. Now assume the vortex changes to a localized spot distribution conserving total vorticity and angular momentum. Because of the conservation of energy, the system cannot become the constant value vorticity sector described above since the sector has larger energy. Instead, there must be some nontrivial distribution of the vorticity inside the spot. The nonaxisymmetric state will therefore have a positive value of entropy and so is the preferred state.

The formation of the localized vorticity is calculated for different values of the circulation $C$, and the maximum energy for the vortex sectors is plotted as the dotted line in Fig. 3. The maximum energy sector becomes a full circular belt, i.e., the same as the symmetrical state, for $C$ greater than 2.9 and the energy becomes smaller than that of the axisymmetric distribution when $C$ is smaller than -0.4 . We therefore find a range of $C$ values where a localized vortex is the preferred state, at least compared to the symmetrical distribution, in the large energy limit.

From the bifurcation analysis, we know that the system has a second-order transition for $C$ between $C_{c}^{\text {lower }}$ and $C_{c}^{\text {upper }}$. To complete the phase diagram in Fig. 3,

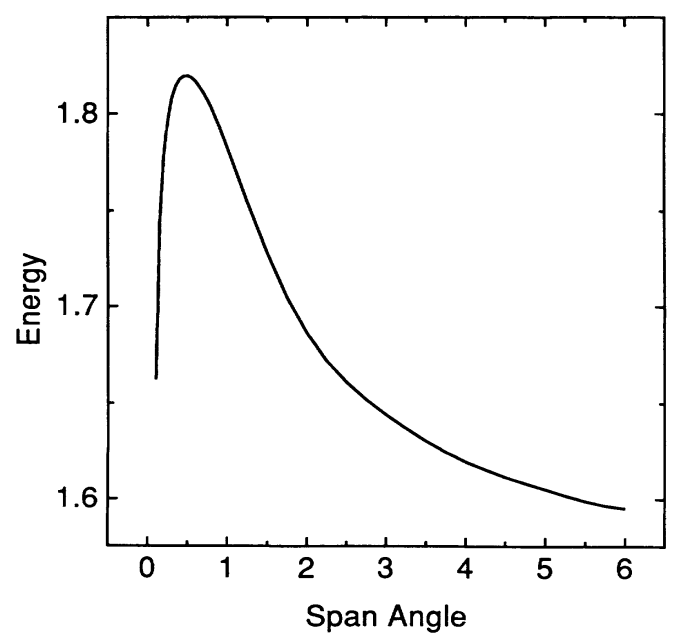

FIG. 4. Typical curve of the energy of the vortex sector versus the span angle. there must be first-order transition lines for $C$ outside $C_{c}^{\text {lower }}$ and $C_{c}^{\text {upper }}$ and in the range mentioned in the previous paragraph. A calculation of the exact position of the line will involve the solution of the two-dimensional partial differential equation to get the distribution of the coherent vortex.

Figure 5 shows the results from both the linear perturbation and vortex sector analysis in the $M-C$ parameter space. The dashed lines are from the linear stability analysis. The solid line is from the zero-temperature variational calculation. In region 2 the axisymmetric solution becomes linearly unstable towards an asymmetrical distribution as the energy is raised. The asymmetric vortex sector will be the thermodynamically preferred state at zero temperature in regions 1,2 , and 3 . In regions 1 and 3 there should be first-order transitions between symmetric and asymmetric states.

We can compare these results with the results of the time evolution simulations of Marcus [6,9]. In the simulations a background velocity distribution, e.g., $v_{\theta}=\beta r^{2} / 3+C^{\prime} / r$, is assumed unchanged with the extra vorticity evolving on top of it. (His $C^{\prime}$ is equal to our $-C$. The $\beta$ term corresponds to a gradient in the Coriolis force which we have not included, although this could easily be done by adding to the Hamiltonian an external potential $-\beta r^{3}$.) The background velocity acts as a shear on the evolving vorticity. One of the main results of the simulations is that for a range of background a single vortex will be stable for $\langle\hat{\sigma}\rangle /\langle\omega\rangle$ greater than a critical value ranging from -0.1 to -0.2 , with $\langle\omega\rangle$ the average vorticity of the spot and $\langle\hat{\sigma}\rangle$ the average value of shear at the vortex. This results holds for different values of $C^{\prime}$ and $\beta$, including the case $\beta=0$. Both the instability analysis and vortex sector approximation in our work also predict the coherent vortex to be the equilibrium state for shear larger than a small negative value. For our geometry and background shear, we find the critical

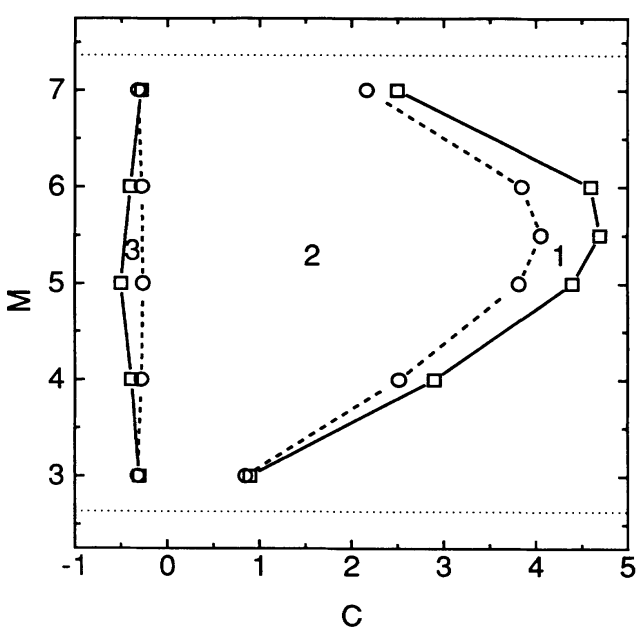

FIG. 5. Phase diagram in the $M-C$ parameter space with $q=1$. The two horizontal dotted lines mark the limiting values of angular momentum $M$. The dashed lines are from the linear bifurcation analysis; the solid lines from the vortex sector calculation. 




FIG. 6. $\epsilon$, the ratio of the length in azimuthal and radial directions for the equilibrium vortex sector, as a function of $C$, and for $Q=2, M=4$, and $q=20$.

ratio $\langle\hat{\sigma}\rangle /\langle\omega\rangle$ to be about -0.43 from the approximate zero-temperature calculation for $\alpha=2 / 3 \pi, Q=2$, and $M=4$, with the ratio becoming smaller for smaller $\alpha$, e.g., -0.24 for the value $\alpha=0.4 / 3 \pi$ which is close to the value in the Marcus simulation.

To investigate the equilibrium shape of the vorticity spot, we define $\epsilon$ as the ratio of the length of the spot in its azimuthal direction to that in the radial. $\epsilon$ is plotted as a function of $C$ in Fig. 6. The sectors are more elongated in the azimuthal direction with increasing circulation $C$. This is reasonable considering that the larger shear stretches the vortex more and more. This result is also consistent with that in Marcus's paper. There a graph shows a similar relation between $\epsilon$ and $\langle\hat{\sigma}\rangle /\langle\omega\rangle$ for a Moor-Saffman ellipse with uniform vorticity $\langle\omega\rangle$.

\section{CONCLUSIONS}

In this paper we have calculated the solution of the exact mean field equations for the statistical mechanics of the Euler equation that respect the infinity of conserved quantities, in two-dimensional axisymmetric geometries resulting from initial conditions consisting of two vorticity levels. Curves are plotted in the energy-entropy and energy-inverse-temperature planes to describe the axisymmetric equilibrium states. Due to the hard cores of the vortices in this description, there is a maximum energy for the vortex system, a significant difference from the point vortex model. For background shears greater than a slightly negative value and not too large to drive the system to the saturated vorticity state, the system is shown to have a continuous bifurcation to a nonaxisymmetric state. The dependence of the bifurcation on the system parameters is accurately calculated by linear stability analysis. This allows an easy way to calculate the dependence of coherent vortex formation on the many system parameters, compared with the numerically intensive time evolution studies. The parameters $M$ and $\alpha$ have large effects on the upper limit, but not the lower limit. For slightly negative and large positive background shears, we find that a discontinuous (first-order) bifurcation to a nonaxisymmetric state must occur, although we have not accurately calculated its location. The properties of the nonaxisymmetric state at high energies (low temperatures) are calculated approximately by a variational scheme.

\section{ACKNOWLEDGMENTS}

The authors acknowledge the support of the National Science Foundation under Grant No. DMR 9013984.
[1] J. Miller, P. B. Weichman, and M. C. Cross, Phys. Rev. A 45, 2328 (1992).

[2] R. Robert and J. Sommeria, J. Fluid Mech. 229, 291 (1991).

[3] G. P. Williams and T. Yamagata, J. Atmos. Sci. 41, 453 (1984).

[4] T. Dowling and A. Ingersoll, J. Atmos. Sci. 46, 3256 (1989).

[5] J. Sommeria, S. D. Meyers, and H. L. Swinney, Nature 331, 689 (1988).

[6] P. S. Marcus, Nature 331, 693 (1988).

[7] M. V. Nezlin, A. Yu. Rylov, A. S. Trubnikov, and A. V. Khutoretskii, Geophys. Astrophys. Fluid Dyn. 52, 211 (1990).

[8] M. V. Nezlin and E. N. Snezhkin, Rossby Vortices, Spiral Structures, Solitons (Springer-Verlag, Berlin, 1993).

[9] P. S. Marcus, J. Fluid Mech. 215, 393 (1990).

[10] J. C. McWilliams, J. Fluid Mech. 146, 21 (1984).

[11] P. Santangelo, R. Benzi, and B. Legras, Phys. Fluids A 1, 1027 (1989).

[12] J. C. McWilliams, J. Fluid Mech. 219, 361 (1990).
[13] W. H. Matthaeus, W. T. Stribling, D. Martinez, S. Oughton, and D. Montgomery, Phys. Rev. Lett. 66, 2731 (1991).

[14] W. H. Matthaeus, W. T. Stribling, D. Martinez, S. Oughton, and D. Montgomery, Physica D 51, 531 (1991).

[15] D. Montgomery, W. H. Matthaeus, W. T. Stribling, D. Martinez, and S. Oughton, Phys. Fluids A 4, 3 (1992).

[16] P. Tabeling, S. Burkhart, O. Cardoso, and H. Willaime, Phys. Rev. Lett. 67, 3772 (1991).

[17] G. F. Carnevale, J. C. McWilliams, Y. Pomeau, J. B. Weiss, and W. R. Young, Phys. Rev. Lett. 66, 2735 (1991).

[18] G. F. Carnevale, J. C. McWilliams, Y. Pomeau, J. B. Weiss, and W. R. Young, Phys. Fluids A 4, 1314 (1992).

[19] R. A. Smith, Phys. Rev. A 43, 1126 (1991).

[20] C. F. Driscoll and K. S. Fine, Phys. Fluids B 2, 1359 (1990).

[21] L. Onsager, Nuovo Cimento Suppl. 6, 279 (1949).

[22] G. Kirchhoff, Lectures on Mathematical Physics, Mechanics (Teubner, Leipzig, 1877).

[23] G. Joyce and D. Montgomery, J. Plasma Phys. 10, 107 (1973). 
[24] Y. B. Pointin and T. S. Lundgren, Phys. Fluids 19, 1459 (1976).

[25] T. S. Lundgren and Y. B. Pointin, Phys. Fluids 20, 356 (1977).

[26] T. S. Lundgren and Y. B. Pointin, J. Stat. Phys. 17, 323 (1977).

[27] R. A. Smith, Phys. Rev. Lett. 63, 1479 (1989).

[28] R. A. Smith and T. M. O'Neil, Phys. Fluids B 2, 2961 (1990).

[29] J. Miller, Phys. Rev. Lett. 65, 2137 (1990).
[30] It is interesting to note that the bifurcation points for the disk are not discovered by a dynamic linear instability analysis, since the growth is always subexpontential for perturbations to axisymmetric solutions in a disk $[\mathbf{R}$. $\mathbf{H}$. Levy, Phys. Fluids 11, 920 (1968)]. This subexponential growth of the $m=1$ mode (the diocotron instability) has been studied by R. A. Smith and M. N. Rosenbluth [Phys. Rev. Lett. 64, 649 (1990)]. Also see C. F. Driscoll and K. S. Fine, Phys. Fluids B 2, 1359 (1990), for experimental results. 